\title{
SNTA1-deficient human cardiomyocytes are associated with increased structural components, calcium handling disorder, and shorter field potential duration
}

\section{Tao Dong ( $\sim$ dong1983tao@qmu.edu.cn )}

Qiqihar Medical University

\section{Yan Zhao}

Qiqihar University

Hai-Feng Jin

Qiqihar Medical College: Qiqihar Medical University

\section{Lei Shen}

Qiqihar Medical College: Qiqihar Medical University

Long-Long Si

Chinese Academy of Sciences Shenzhen Institutes of Advanced Technology

\section{Li Chen}

Chinese Academy of Sciences Shenzhen Institutes of Advanced Technology

\section{Research Article}

Keywords: hESCs, SNTA1-deficient cardiomyocyte, CRISPR-Cas9, calcium homeostasis

Posted Date: January 28th, 2022

DOI: https://doi.org/10.21203/rs.3.rs-1255102/v1

License: (c) (i) This work is licensed under a Creative Commons Attribution 4.0 International License.

Read Full License 


\section{Abstract \\ Background}

a-1-syntrophin (SNTA1), a protein encoded by SNTA1, is highly expressed in human cardiomyocytes. Mutations in SNTA1 are associated with arrhythmia and cardiomyopathy. Previous research on SNTA1 has been based on nonhuman cardiomyocytes. Our study was designed to identify phenotype of SNTATdeficient using human cardiomyocytes.

\section{Methods}

SNTA1 was knocked out in H9 cell line using CRISPR-Cas9 system. H9SNTA1KO cells were then induced to differentiate into cardiomyocytes using small molecule inhibitors. The phenotypic discrepancies associated with SNTA1-deficient cardiomyocytes were investigated.

\section{Results}

SNTA1 was truncated in PH1 domain by a stop codon (TGA) using CRISPR-Cas9 system. SNTA1-deficient did not affect the pluripotency of H9SNTA1KO, and they retain their in vitro ability to differentiate into cardiomyocytes. However, H9SNTA1KO derived cardiomyocytes exhibited increased structural components, weak calcium transient intensity, low levels of calcium in sarcoplasmic reticulum, lower cardiac contractility, and shorter field potential duration. Early treatment of SNTA1-deficient cardiomyocytes with ranolazine improved the calcium transient intensity and cardiac contractility.

\section{Conclusions}

SNTA1-deficient cardiomyocytes can be used to research the etiology, pathogenesis, and potential therapies for myocardial diseases. The SNTA1-deficient cardiomyocyte model suggests that the maintenance of cardiac calcium homeostasis is a key target in the treatment of myocardial-related diseases.

\section{Introduction}

SNTA1 becomes an important signaling scaffold protein between the extracellular matrix and the intracellular cytoskeleton by connecting with dystrophin-associated protein complex (DAPC) [1]. The point mutations of SNTA1 can cause long QT syndrome [2-3], Brugada syndrome [4], sudden infant death syndrome [5], atrial fibrillation [6], etc. The SNTA1 binds the motif of PDZ domain of Nav1.5. Nav1.5 is an important type of cardiac voltage-gated sodium channels. SNTA1 plays a critical auxiliary role in the correct subcellular localization, expression, and function of Nav1.5. SNTA1 is involved in the regulation of membrane volume on Kir2.1and Kir2.2 channels [7]. In Snta1 knockout mice, left ventricular posterior wall 
thickening and abnormal myocardial performance index (MPI) has been reported, which indicates the presence of myocardial hypertrophy in the knockout mice [8].

At present, the results of research on SNTA1 are from nonhuman cells, such as $\mathrm{CHO}$ cells, $\mathrm{H} 9 \mathrm{C} 2$ cells, Xenopus oocytes, etc. A large number of patient cardiomyocytes can be derived from patients' iPSCs. Patients' cardiomyocytes were studied for abnormal phenotypes, however, there are a lot of differences between patients and normal donors in terms of the genetic background. Different genetic background hampered the further research in patients' iPSCs. With the widespread applications of gene editing technologies in eukaryotic cells [9-11], genetically modified human embryonic stem cells (hESCs) establishment help to resolve the impediment of the different genetic background [12-13]. In order to investigate the phenotype of SNTA1-deficient cardiomyocytes, SNTA1-deficient cardiomyocytes induction from the CRISPR-Cas9-modified hESCs were researched, and then compared with the phenotype of wild type (WT) cardiomyocytes under the same genetic background. This research provides an example of using human cell to study the phenotype of cardiomyocytes caused by the SNTA1 knockout, meanwhile, providing a case that the exploration of the mechanism of pathogenesis of gene knockout using human cardiomyocytes.

\section{Methods}

\section{Embryonic stem cell culture and the design for SNTA1 knockout}

The $\mathrm{H} 9$ embryonic stem cell was purchased from WiCell Research Institute Inc. The H9 cells were cultured in E8 medium, and when the cells reached $80 \%$ confluence, they were digested by 0.5 mM EDTA. Using the CRISPR-Cas9 system, Zhang Lab web tools were used to design single guide RNA (sgRNA) targeting SNTA1. The sgRNA targeting site was used to select public exons close to start codon: we selected exon 2 design sgRNA (5'-attggcaggacag-3') and confirmed deletion by western blots.

\section{Embryoid body (EB) formation test and monolayer differentiation}

With 0.5 mM EDTA, $6 \times 10^{6}$ cells were suspended in PSCeasy Medium (Cellapy, China) with $2 \mu \mathrm{M}$ thiazovivin (TZV) and plated in a 6-wells plate without Matrigel for 4 days, then the EBs were cultured in RPMI medium 1640 with $20 \%$ knockout serum replacement (KSR) in the Matrigel-coated plate. The embryonic stem cells were induced to differentiate into cardiomyocytes using CardioEasy Kit (Cellapy, China) in the Matrigel-coated plate for monolayer differentiation.

\section{RNA-sequencing (RNA-seq) analysis and qRT-PCR}

After RNA was extracted from SNTA1-deficient cardiomyocytes (KO) and WT, RNA-seq was analyzed by BGI Tech. Solutions Co., Ltd. (Liuhe, China). Total cellular RNA was extracted with TRIzol (Invitrogen, USA) and treated with DNase I (Beyotime, China) for approximately $30 \mathrm{~min}$ at $37^{\circ} \mathrm{C}$ to eliminate DNA contamination. RNA was reverse transcribed using the Prime-Script TM reverse transcription system (TaKaRa, Japan). Relative gene expression levels were examined by qRT-PCR using the iCycler iQ5 (Bio- 
Rad, USA) with TB Green ${ }^{\text {TM }}$ Premix Ex Taq ${ }^{\text {TM }}$ II (Takara, Japan). The relative quantification was calculated according to the $\mathbb{X C T}$ method.

\section{Flow cytometry}

The cells were digested with $0.5 \mathrm{mM}$ EDTA to prepare single-cell suspensions, and then incubated with the antibody for $30 \mathrm{~min}$ in PBS at room temperature (RT). The samples were detected by Flow cytometer (Beckman, EPICS XL) and the results were analyzed using the Flow Jo VX software.

\section{Immunofluorescent staining}

Cells were fixed in $4 \%$ paraformaldehyde for 30 min, washed three times in PBS, permeabilized with $0.3 \%$ Triton X-100 for 10 min at RT, and blocked in 3\% BSA for 30 min at RT. Then the cells were incubated with the primary antibody for $24 \mathrm{~h}$ at $4^{\circ} \mathrm{C}$, washed three times in PBS, and then the cells were incubated with the secondary antibody and DAPI (100 nM) for $1 \mathrm{~h}$ at RT. The cells were subsequently washed again three times in PBS, and imaged with the confocal microscopy (Leica, TCS5 SP5).

\section{Calcium transient assay and caffeineevoked calcium release test}

The cardiomyocytes were plated on $35 \mathrm{~mm}$ confocal dish loaded with $4 \mu \mathrm{M}$ Fluo-4 AM (Yeasen, China) and incubated at $37^{\circ} \mathrm{C}$ for 20 min in PBS (Servicebio, China) containing $0.04 \%$ Pluronic F-127 (Yeasen, China). PBS was changed to the Cardiomyocytes Maintenance Medium (Cellapy, China). Loaded samples were transferred under a TCS-SP5-RS confocal microscope (Leica, Germany). Laser emission at $488 \mathrm{~nm}$ was used for stimulation and emitted fluorescence at $530 \mathrm{~nm}$ was acquired. Samples were then stimulated with freshly prepared solution of caffeine $(20 \mathrm{mM})$, and emitted fluorescence acquired to record transient alteration in cytosolic calcium levels.

\section{Contractility measurement and microelectrode array test}

The contractility of cardiac myocytes was measured according to the protocols reported previously [1415]. The electrical activity of cardiac myocytes was detected using Maestro Multiwell Microelectrode Array (MEA). The experimental was carried out according to the protocol of micro electrode array.

\section{Statistical methods}

The data of measurement and count were all presented as mean \pm standard deviation. The difference between two groups was analyzed by one-tail or two-tail student's $t$ test, and the rate was compared by Fisher's Exact test, three groups of data and more were analyzed using single-factor or two-factor analysis of variance, followed by Tukey multiple comparison test. The confidence interval was $95 \%$, ${ }^{*}<$ $0.05, * * P<0.01, * * * P<0.001, * * * * P<0.0001$, representing four levels of statistical significance.

\section{Results}


We selected the second exon of SNTA1, corresponding to the pleckstrin homology 1 (PH1) domain, as the target site of sgRNA (Fig.1A). The result of DNA sequencing indicated that an adenine nucleotide was inserted before the protospacer adjacent motif (PAM) region after gene editing (Fig.1B). A stop codon (TGA) appeared at the amino acid position 149 in the SNTA1 and terminated the SNTA1 protein prematurely in the $\mathrm{PH} 1$ domain. The rate of SNTA1 knockout were evaluated using the Synthego analysis sequencing result (Fig.1C). Immunofluorescence staining for pluripotency was performed on H9SNTA1KO. Both SSEA4 and NANOG were positive with H9SNTA1KO. (Fig.1D). The expression of pluripotent markers SOX2, DPPA4, OCT-4, and NANOG in H9SNTA1KO were similar to WT using qRT-PCR analysis (supplementary data Fig.1A). The karyotype analysis of H9SNTA1KO was normal (46, XX) (supplementary data Fig.1B). Also, western blots confirmed H9SNTA1KO to be deficient in SNTA1 (supplementary data Fig.1C).

\section{H9SNTA1KO differentiated into cardiomyocytes}

Schematic of the H9SNTA1KO which were induced into cardiomyocytes using small molecule inhibitors (Fig.2A). The image of H9SNTA1KO induced using small molecule inhibitors from stage 1 through 3 (Fig.2B1, B2, B3, respectively). We noticed the beating of $\mathrm{KO}$ on the $10^{\text {th }}$ day of cardiac differentiation (Fig.2B4). KO were enriched with metabolic selection using glucose-free medium containing lactate. The KO layer was formed after cell density adjustment (Fig.2C). In order to identify the kind of differentiated cells, we stained the cells with TNNT2 and a-actinin cardiomyocytes markers. The result showed the beating cells, which differentiated from H9SNTA1KO, were positive for TNNT2 and a-actinin (Fig.2D). We examined the expression of the TNNT2 in WT and KO before metabolic selection using flow cytometry assay. The results indicated that H9SNTA1KO were capable of differentiating into cardiomyocytes (Fig.2E). MYL2, a specific marker of ventricular muscle, was detected in WT and KO after metabolic selection using flow cytometry assay. The results showed that the H9SNTA1KO had a normallydifferentiated cardiomyocyte subtype (Fig.2F).

\section{Structural components increased in KO}

In order to study the phenotype of KO, The RNA of KO was analyzed by RNA-seq after being cultured on the $30^{\text {th }}$ day. The data were analyzed using KEGG (Kyoto Encyclopedia of Genes and Genomes) enrichment to discover the gene activation of hypertrophy-related pathway in KO (supplementary data Fig.2A, B, C, respectively). The main structural components of $\mathrm{KO}$ were detected by qRT-PCR, including TNNT2, MYH6, MYH7, MYL2囚MYL7\etc. The results suggested that the structural components involved in contraction of $\mathrm{KO}$ were elevated at the transcription level (Fig.3A). For instance, the results indicated that the expression levels of TNNT2, MYL2, and a-actinin in KO were higher than those in WT cultured on the $45^{\text {th }}$ day (Fig.3B, C, D, respectively). The expression of MYL2 was detected using western blotting technique. The results showed the expression was higher in KO (supplementary data Fig.2D). The p$\mathrm{Ca}^{2+} /$ calmodulin-dependent protein kinase II (p-CaMKII) level, which is usually with abnormal calcium handling, was detected by western blots. The results confirmed that $\mathrm{p}$-CaMKII levels increased in KO (supplementary data Fig.2E). 


\section{The KO had normal diameter and shorter field potential duration (FPD)}

In general, the diameter of a cell is affected by structural components increasing. Comparing the diameters of KO to those of WT used flow cytometry forward scatter (FSC). The results showed the diameters of KO were not different from those of the WT (Fig.4A, B, C, respectively) on the $15^{\text {th }}, 35^{\text {th }}$, and $45^{\text {th }}$ day. The mutations of SNTA1 were usually involved in the variation of QT interval. SNTA1 was thought to be the long QT syndrome (LQT) type 12 gene. A variation of QT interval was simulated by the FPD using MEA. The FPD of KO were measured with the MEA (Fig.4D, E, respectively). The results confirmed that the FPD values of the KO were shorter than those of WT.

\section{The KO exhibited calcium transient abnormality}

The abnormalities in structural components of cardiomyocytes are usually caused by the abnormality of calcium handling. We carried out calcium transient test in WT and KO (Fig.5A). The space-averaged calcium transients displaying the parameters measured for analysis of calcium handling (Fig.5B). The results showed that the peak value of calcium release was remarkably decreased in $\mathrm{KO}$ (Fig.5C). The time to peak and the decay time of calcium transient were shorter in KO (Fig.5D, E, respectively). In addition, the contraction duration of $\mathrm{KO}$ was shorter (Fig.5F). Furthermore, the beating rate of $\mathrm{KO}$ was increased (Fig.5G). All of these results confirmed that intracellular calcium handling of $\mathrm{KO}$ was abnormal.

\section{Abnormal caffeineevoked $\mathrm{Ca}^{2+}$ release in $\mathrm{KO}$}

There was a disorder of intracellular calcium handling in $\mathrm{KO}$ with the peak of calcium release decreased. The peak of intracellular calcium release is directly related to the sarcoplasmic reticulum (SR) calcium storage in cardiomyocytes. Therefore, we conducted caffeine-evoked $\mathrm{Ca}^{2+}$ release test on the KO (Fig.6A). The results indicated that the peak of caffeine-evoked calcium release was decreased in KO (Fig.6B) with the time of peak and Decay time from $50 \%$ peak being shorter (Fig.6C, D, respectively). The calcium ion level in SR was decreased in the KO compared with the WT. The release of calcium ion from SR is related to the contractility of cardiomyocytes. There was a decrease SR calcium level in the $\mathrm{KO}$, the contractility of $\mathrm{KO}$ was detected. The contractility of cardiac myocytes was measured using quantification of muscle contraction method (Fig.6E). The results showed that the amplitude of contraction was decreased in KO compared with WT (Fig.6F, G, respectively).

\section{Impairment of calcium handling in KO}

We found there was an abnormal calcium handling in $\mathrm{KO}$. We detected the expression of some genes involved in the calcium by qRT-PCR. The result indicated that SERCA2a, BIN1, and CASQ2 were abnormal at transcriptional levels (Fig.7A). The SERCA2a, BIN1, and CASQ2 levels were further detected using western blots. The results showed that expression level of SERCA2a and CASQ2 decreased, while BIN1 increased (Fig.7B, C, D, respectively). Overall, there was impairment in calcium handling in KO.

\section{Early application of ranolazine improving the calcium handling of $\mathrm{KO}$}


The application of ranolazine significantly promoted the intracellular calcium handling, prevented intracellular calcium overload, and increased calcium loading in SR during diastole. Ranolazine $(10 \mu \mathrm{M})$ was added on the $20^{\text {th }}$ day cultured WT and KO for $24 \mathrm{~h}$, respectively. Then, the calcium transient test was performed on the KO (Fig.8A). The result showed the time to peak in $\mathrm{KO}$ treated with ranolazine was shorter compared with no-treatment (Fig.8B). The peak of calcium transient test increased in KO treated with ranolazine compared with no-treatment (Fig.8C). The contraction duration became shorter in KO treated with ranolazine compared with no-treatment (Fig.8D). The beating rate was elevated in $\mathrm{KO}$ treated with ranolazine compared with no-treatment (Fig.8E). The results of calcium transient test of WT treated with ranolazine were in the supplementary data Fig.3A-E. The results showed that early application of ranolazine elevated the peak of calcium release in $\mathrm{KO}$. We speculated the increasing calcium transient would ameliorate the contraction force. Hence, we detected the contraction force of KO treated with ranolazine. The result showed the contractility force was increased in $\mathrm{KO}$ treated with ranolazine (Fig.8F, $\mathrm{G} \otimes$ respectively). All the results demonstrated that ranolazine improved calcium release from SR in KO at early application.

\section{Discussion}

SNTA1 is highly expressed in human heart. As a scaffold protein, SNTA1 binds to the C-terminal of sodium voltage-gated channel alpha subunit 5 (SCN5A) and played a vital regulatory role in SCN5A [5]. Recently, it has been reported that SNTA1 interacted with the N-terminal domain of SCN5A [7]. The MPI was elevated in Snta 1 knockout mice; the results indicated that Snta1 knockout mice had left ventricular dysfunction and hypertrophy [8]. Our cell model highlighted the indispensable role of SNTA1 in the cardiomyocyte of calcium handing (Fig. 9). In Human Gene Mutation Database (HGMD), diseases caused by SNTA1 point mutation, including LQT, Brugada, sudden infant death syndrome, cardiomyopathy, atrial fibrillation, etc., were recorded. For elucidating the functions of SNTA1, CHO, 293T, and mice were used as research model. The major limitation is the transition from animal to human studies due to the species differences. The results of animal experiments do not completely replace human experiments. In general, it is difficult to obtain a large number of human cardiomyocytes. The hESCs differentiated into cardiomyocytes provide an opportunity to investigate cardiac development and disease as well as providing a platform to perform drug and toxicity tests.

Currently, there are three methods for myocardial differentiation, including EB differentiation method, heart organoids method, and small molecule inhibitors differentiation method. Every method has own characteristics. Traditional spontaneous 3D method by EB differentiation of hESCs into cardiomyocytes is inefficient. It is not easy to obtain a single-type cardiomyocyte. Heart organoids technique simulates the whole organ and is not conducive to the study of the ventricular muscle alone [16]. A single type of human ventricular-like muscle can be obtained using small molecule inhibitors, and the ventricular-like muscle can be purified using metabolic purification method in vitro [17]. The H9SNTA1KO was established using the CRISPR-Cas9 system, and then induced the H9SNTA1KO to differentiate into 
cardiomyocytes using small molecule inhibitors. The result confirmed that SNTA1-deficient did not affect the differentiation of cardiomyocytes and subtypes of cardiomyocyte using flow cytometry assay.

Furthermore, Transcriptomic discrepancies were investigated between KO and WT using RNA-seq, after cardiomyocytes were purified using the metabolic method [18]. Analysis of RNA-seq data revealed that KO had activation of genes associated with hypertrophy. qRT-PCR and immunofluorescence quantitative analysis on genes associated with the structure of cardiomyocytes were performed. Thus, the activation of genes associated with cardiac hypertrophy was confirmed. The results suggested that the expression of structural genes was enhanced on the 45th day of cardiac differentiation. The KO exhibited hypertrophy phenotype like the Snta 1-knockout animal's cardiac muscle. We also found that TNNT2, MYH7, MYH6, and MYH7/MYH6 increased in transcriptional level on the 45th day of cardiac differentiation. In the HGMD, point mutations in SNTA1 were associated with arrhythmia. SNTA1 encodes the a1-syntrophin that is responsible for LQT12 in an autosomal-dominant manner (SNTA1 is a disputedevidence or limited-evidence gene in genotype-phenotype correlations [19]). In common, the disease phenotypes of $\mathrm{KO}$ were more serious than the other point mutation. The disease phenotype was most serious in the knockout cell or knockout animal, but the Snta 1-knockout mice did not exhibit any arrhythmia in Kim's paper [8]. When human cardiomyocytes were used, the arrhythmia might be exhibited. We compared the FPD of KO to the WT using MEA. The parameters of FPD in MEA simulates to the QT intervals of electrocardiograph. With the results indicating that FPD of KO was shorter than that of WT. SNTA1 point mutations caused complex genetic arrhythmias, including LQT, Brugda, and sudden infant death. SNTA1 interacted with the $\mathrm{N}$ - and C-terminal of SCN5A [2], and also adjusted the density of human Kir2.1, Kir2.2 in cardiomyocyte membrane [7]. SNTA1 is also involved in the dystrophin/utrophin protein complex and help the target protein to the right location in cardiomyocyte [20]. In our research, the mutation we created terminated SNTA1 at the 149 amino acid position, resulting in the termination of SNTA1 at the PDZ domain The short QT syndrome caused by SNTA1 deficiency may be associated with channel dysfunction.

The diameter of KO was compared with the WT on the 15th, 35th, and 45th day of cardiac differentiation. The result that the diameter of KO was not different from the WT. SNTA1 binds to the dystrophin glycoprotein complex through its PH2 and SU domains. SNTA1 also binds to the cytoskeleton protein, such as actin, microfilament, and microtubule ${ }^{[21]}$. The organization of cytoskeleton in KO may be disrupted by SNTA1 deficiency, but the diameter of KO did not be influenced. However, we only harvested ventricular-like muscle cells with no morphological maturity. We speculated that the immature morphological features of the cardiomyocytes influenced the result of diameter measurement.

The function of cardiomyocytes is precisely dependent on the calcium handling. Calcium is a critical intracellular signaling molecules, which mediates various biological processes, including excitationcontraction coupling (EC), enzyme activity, gene transcription, and cell death [22-23]. Calcium is necessary for heart contraction. Particular attention was paid to the transport and storage of calcium in cardiomyocytes since an abnormal calcium handling plays a key role in the pathogenesis of cardiomyopathy and arrhythmia [24-25]. The EC of cardiomyocytes requires the coordinated transport of 
calcium in and out cell. In the physical process of myocardial contraction, Nav1.5, which is a sodium channel on the membrane of ventricular myocytes, is activated by an electrical stimulation from the pacemaker, and then Nav1.5 channel opens and the extracellular sodium flows into the cells forming an ascending branch of action potential. The CACNA1C in T-tubules induced the extracellular calcium influx into cell (calcium sparks) by depolarization of membranes, and calcium influx activated the RyR2 on the $\mathrm{SR}$, with the calcium in SR released through the RyR2 into the cytoplasm. Elevated free calcium in the cytoplasm causes myofilament contraction. In the process of calcium-induced-calcium-release (CICR), there are three proteins to help maintain the function of the T-tubule [26]. The first protein is junctophilin 2 (JPH2), which is the major structural protein in cardiomyocytes between the T-tubules and the SR. It drags the T-tubules closer to SR to form the junctional membrane complex, facilitating CICR [27-29]. The CICR is a critical procedure of EC in ventricular myocytes. The second protein is caveolin 3 (CAV3), a member of caveolin protein family that contribute to the formation of caveolae and provide microdomains for a variety of functional proteins in T-tubules [30-32]. The third protein is bridging integrator 1 (BIN1), one of the membrane scaffolding proteins, which interact between BAR domain and phospholipid acid in the cell membrane to deform the membrane bilayer. BIN1 is not only involved in the formation of the T-tubules, but also transports the calcium voltage-gated channel subunit alpha1 C (CACNA1C) to the cell membrane. A decrease in the expression of BIN1 resulted in decrease in trafficking of CACNA1C to the Ttubules [33]. Therefore, rescuing diminished cardiac BIN1 expression could both rescue CACNA1C trafficking and restore normal T-tubule membrane morphology [34]. BIN1 could become a therapeutic target for heart failure [35-36]. When the cytoplasmic free calcium is elevating, calcium binds to troponin $\mathrm{C}$ and triggers contraction, and cause the concentrations of intracellular free calcium to elevate from $0 \sim 100 \mathrm{nM}$ to $\sim 1 \mu \mathrm{M}$ compared with the diastole period [37]. After contraction, SERCA2a is a macromolecular complex on the SR that reuptakes the free calcium back into the SR (intracellular calcium pool), facilitating cardiac relaxation. A small portion of cytosolic calcium is extruded by the NCX (sodium-calcium exchanger), and only a small level of released calcium enters the mitochondria [38].

The increased structural components are usually associated with abnormal calcium handling in cardiac myocytes [39-40]. In our cell model, there was an increase of structural components in KO. Calcium transient test was performed on $\mathrm{KO}$, the results showed that the peak value of $\mathrm{KO}$ was lower than the WT(Fig. 5). The peak value of calcium transient is related to the level of calcium in the SR. The calcium load in SR was measured using caffeine-evoked calcium release test to elucidate the effect of SR function. These results showed that the SR of calcium loading decreased in KO (Fig. 6). The contraction of KO was lower than the WT in vitro, which is consistent with the result of calcium transient test.

The calcium release is related to the calcium loading in the SR. CASQ2, ASPH, and TRDN form complexes, which is involved in the SR calcium loading [41-43]. We assessed the genes involved in calcium loading and calcium handling. The results demonstrated that the expression of CASQ2, BIN1, and SERCA2a in both the transcriptional and protein levels were significant. The expression level of CASQ2 in KO was lower than WT. CASQ2 is a calcium-binding protein, which exists in the SR of cardiac muscle. It is low-affinity and high-capacity for binding calcium. It binds to 40-50 calcium ions through 6070 negatively charged amino acid residues [44-45]. CASQ2 is considered to be an essential protein for 
the storage and release of calcium in the SR [46]. The point mutations in CASQ2 can lead to ventricular tachycardia [47-48], cardiomyopathy [49], catecholamine ventricular tachycardia [50], and the other heart-related diseases. The Casq2knockdown mice only showed obvious SR calcium leak [51] and displayed early mortality [52]. The decrease of CASQ2 expression in KO leaded to the decrease of calcium loading in the SR during diastole. We speculated that decreased of CASQ2 expression maybe disturbed by the disorder of calcium handing. The specific mechanism will be verified by follow-up research. BIN1 was another scaffold protein under the cellular membrane. We speculated the high expression of BIN1 was a kind of cellular compensatory protection. In addition, the expression of SERCA2a was decreased in $\mathrm{KO}$, which showed that the SR weakly reuptakes free calcium from cytoplasm. There was a decrease expression of CASQ2, an increase expression of BIN1, and a decrease expression of SERCA2a in the KO. There was an abnormal calcium homeostasis in our cell model.

A decrease SR calcium loading of cardiomyocytes has no effective remedy currently. The metabolic support therapy could help to relieve the symptoms of cardiomyocyte diseases [53-55]. The metabolic support efforts alleviate the abnormal energy supply of hypertrophic, promote the survival rate, and improvement of myocardial cell functions [56]. Ranolazine inhibited the action of late sodium current, improved diastolic calcium overload, and the relaxation of ventricular myocytes [57-58]. It also enhanced the NCX extroversion mode, indirectly promoted the intracellular calcium excretion, and reduced high free intracellular calcium in diastole [59-60]. We utilized $10 \mu \mathrm{M}$ ranolazine to improve phenotype of $\mathrm{KO}$ on the 20th day of cardiac differentiation. The results showed that the peak of calcium transient amplitude and the contraction force increased in KO. Overall, the early application of ranolazine improved the phenotype of $\mathrm{KO}$.

\section{Limitation}

The cardiomyocytes we obtained had no T-tubules structure and that was unlike the mature cardiomyocytes. Our research was only performed in the two-dimensional (2D) cell culture in vitro.

\section{Conclusions}

In the study, human SNTA1-knockout cell model was established using CRISPR-Cas9 system. The cell model can be used to study the damage of myocardial structure and abnormal function caused by the SNTA1 deficiency in vitro. It provides a disease model for intracellular calcium homeostasis of cardiomyocytes study. It confirms that abnormal intracellular calcium handling was the core disease mechanism of SNTA1-deficient cardiomyocytes. This suggests that the strategy of maintenance of the intracellular calcium homeostasis is a key target in treatment of SNTA1-deficient myocardial diseases.

\section{Abbreviations}




\begin{tabular}{|c|c|}
\hline Symbol & Full Name \\
\hline SNTA1 & a-1-syntrophin \\
\hline hESCs & human embryonic stem cells \\
\hline H9SNTA1KO & SNTA1-deficient hESCs \\
\hline WT & Wilde type \\
\hline KO & SNTA1-deficient cardiomyocytes \\
\hline PAM & protospacer adjacent motif \\
\hline $\begin{array}{l}\text { CRISPR- } \\
\text { Cas9 }\end{array}$ & $\begin{array}{l}\text { clustered regularly interspaced short palindromic repeats/CRISPR-associated protein } \\
9\end{array}$ \\
\hline TNNT2 & troponin T2, cardiac type \\
\hline SSEA4 & stage-specific embryonic antigen- 4 \\
\hline NANOG & Nanog homeobox \\
\hline SOX2 & SRY-box transcription factor 2 \\
\hline DPPA4 & developmental pluripotency associated 4 \\
\hline OCT-4 & POU class 5 homeobox 1 \\
\hline MYL2 & myosin light chain 2 \\
\hline KEGG & Kyoto Encyclopedia of Genes and Genomes \\
\hline MYH6 & myosin heavy chain 6 \\
\hline MYH7 & myosin heavy chain 7 \\
\hline MYL7 & myosin light chain 7 \\
\hline p-CaMKII & $\mathrm{p}-\mathrm{Ca}^{2+} /$ calmodulin-dependent protein kinase II \\
\hline CaMKII & $\mathrm{Ca}^{2+} /$ calmodulin-dependent protein kinase II \\
\hline FPD & short field potential duration \\
\hline EC & excitation-contraction coupling \\
\hline SR & sarcoplasmic reticulum \\
\hline CACNA1C & calcium voltage-gated channel subunit alpha1 C \\
\hline SERCA2a & ATPase sarcoplasmic/endoplasmic reticulum $\mathrm{Ca}^{2+}$ transporting 2 \\
\hline BIN1 & bridging integrator 1 \\
\hline CASQ2 & CASQ2 \\
\hline
\end{tabular}




\begin{tabular}{|ll|}
\hline RyR2 & ryanodine receptor 2 \\
\hline CICR & calcium-induced-calcium-release \\
\hline JPH2 & junctophilin 2 \\
\hline CAV3 & caveolin 3 \\
\hline NCX & sodium-calcium exchanger \\
\hline ASPH & aspartate beta-hydroxylase \\
\hline TRDN & triadin \\
\hline
\end{tabular}

\section{Declarations}

\section{Funding}

We gratefully acknowledge funding support from the Doctoral Foundation of Qiqihar Medical University Nos.QMSI2021B-07, National Natural Science Foundation of China Nos. 32101694.

\section{Competing interests}

The authors declare that they have no competing interests.

\section{Ethics approval}

Not applicable

\section{Consent to participate}

Not applicable

\section{Consent for publication}

Not applicable

\section{Availability of data and material}

All data transparency

\section{Code availability}

The RNA-seq data associated with this manuscript can be inquired from biosys.bgi.com (Username: F20FTSNCWLJ4149_PEOxzmR).

\section{Authors' contributions}


DT conceived the idea and designed the experiments; DT and ZY performed the cell experiments and data analysis. DT and SL performed the manuscript preparation. JHF is responsible for the collection and assembly of data. ZY contributed to the molecular experiments. LC contributed to the function analysis. SLL has been helping with revisions. All authors read and approved the final manuscript.

\section{Acknowledgements}

We thank Feng Lan Professor from National Center for Cardiovascular Diseases to provide hESCs and the method of obtaining cardiomyocytes from hESCs. We thank Yong-Ming Wang Professor from School of Life Sciences, Fudan University to provide the CRISPR-Cas9 plasmid. We are grateful to Hong-Feng Jiang Professor from Beijing Laboratory for Cardiovascular Precision Medicine for editing the article.

\section{References}

1. Sealock R, Butler MH, Kramarcy NR, Gao KX, Murnane AA, Douville K, Froehner SC. Localization of dystrophin relative to acetylcholine receptor domains in electric tissue and adult and cultured skeletal muscle. J Cell Biol. 1991 Jun;113(5):1133-44. doi: 10.1083/jcb.113.5.1133.

2. Ueda K, Valdivia C, Medeiros-Domingo A, Tester DJ, Vatta M, Farrugia G, Ackerman MJ, Makielski JC. Syntrophin mutation associated with long QT syndrome through activation of the nNOS-SCN5A macromolecular complex. Proc Natl Acad Sci U S A. 2008 Jul 8;105(27):9355-60. doi: 10.1073/pnas.0801294105.

3. Wu G, Ai T, Kim JJ, Mohapatra B, Xi Y, Li Z, Abbasi S, Purevjav E, Samani K, Ackerman MJ, Qi M, Moss AJ, Shimizu W, Towbin JA, Cheng J, Vatta M. alpha-1-syntrophin mutation and the long-QT syndrome: a disease of sodium channel disruption. Circ Arrhythm Electrophysiol. 2008 Aug;1(3):193-201. doi: 10.1161/CIRCEP.108.769224.

4. Brion M, Blanco-Verea A, Sobrino B, Santori M, Gil R, Ramos-Luis E, Martinez M, Amigo J, Carracedo A. Next generation sequencing challenges in the analysis of cardiac sudden death due to arrhythmogenic disorders. Electrophoresis. 2014 Nov;35(21-22):3111-6. doi:10.1002/elps.201400148.

5. Cheng J, Van Norstrand DW, Medeiros-Domingo A, Valdivia C, Tan BH, Ye B, Kroboth S, Vatta M, Tester DJ, January CT, Makielski JC, Ackerman MJ. Alpha1-syntrophin mutations identified in sudden infant death syndrome cause an increase in late cardiac sodium current. Circ Arrhythm Electrophysiol. 2009 Dec;2(6):667-76. doi:10.1161/CIRCEP.109.891440.

6. Husser D, Ueberham L, Hindricks G, Büttner P, Ingram C, Weeke P, Shoemaker MB, Adams V, Arya A, Sommer P, Darbar D, Roden DM, Bollmann A. Rare variants in genes encoding the cardiac sodium channel and associated compounds and their impact on outcome of catheter ablation of atrial fibrillation. PLoS One. 2017 Aug 24;12(8):e0183690. doi: 10.1371/journal.pone.0183690.

7. Matamoros M, Pérez-Hernández M, Guerrero-Serna G, Amorós I, Barana A, Núñez M, Ponce-Balbuena D, Sacristán S, Gómez R, Tamargo J, Caballero R, Jalife J, Delpón E. Nav1.5 N-terminal domain 
binding to a1-syntrophin increases membrane density of human Kir2.1, Kir2.2 and Nav1.5 channels. Cardiovasc Res. 2016 May 15;110(2):279-90. doi: 10.1093/cvr/cvw009.

8. Kim MJ, Whitehead NP, Bible KL, Adams ME, Froehner SC. Mice lacking $\alpha$-, $\beta 1$ - and $\beta 2$-syntrophins exhibit diminished function and reduced dystrophin expression in both cardiac and skeletal muscle. Hum Mol Genet. 2019 Feb 1;28(3):386-395. doi: 10.1093/hmg/ddy341.

9. Jinek M, Chylinski K, Fonfara I, Hauer M, Doudna JA, Charpentier E. A programmable dual-RNAguided DNA endonuclease in adaptive bacterial immunity. Science. 2012 Aug 17;337(6096):816-21. doi: 10.1126/science.1225829.

10. Cong L, Ran FA, Cox D, Lin S, Barretto R, Habib N, Hsu PD, Wu X, Jiang W, Marraffini LA, Zhang F. Multiplex genome engineering using CRISPR/Cas systems. Science. 2013 Feb 15;339(6121):819-23. doi: $10.1126 /$ science. 1231143 .

11. Koblan LW, Erdos MR, Wilson C, Cabral WA, Levy JM, Xiong ZM, Tavarez UL, Davison LM, Gete YG, Mao X, Newby GA, Doherty SP, Narisu N, Sheng Q, Krilow C, Lin CY, Gordon LB, Cao K, Collins FS, Brown JD, Liu DR. In vivo base editing rescues Hutchinson-Gilford progeria syndrome in mice. Nature. 2021 Jan;589(7843):608-614. doi: 10.1038/s41586-020-03086-7.

12. Xie Y, Wang D, Lan F, Wei G, Ni T, Chai R, Liu D, Hu S, Li M, Li D, Wang H, Wang Y. An episomal vectorbased CRISPR/Cas9 system for highly efficient gene knockout in human pluripotent stem cells. Sci Rep. 2017 May 24;7(1):2320. doi: 10.1038/s41598-017-02456-y. Erratum in: Sci Rep. 2018 Dec 12;8(1):17900.

13. Dong T, Zhang S, Chang Y, Bai R, Jiang Y, Ma S, Li Y, Jiang HF, Lu WJ. The establishment of a homozygous SNTA1 knockout human embryonic stem cell line (WAe009-A-50) using the CRISPR/Cas9 system. Stem Cell Res. 2021 Mar;51:102196. doi:10.1016/j.scr.2021.102196.

14. Sala L, van Meer BJ, Tertoolen LGJ, Bakkers J, Bellin M, Davis RP, Denning C, Dieben MAE, Eschenhagen T, Giacomelli E, Grandela C, Hansen A, Holman ER, Jongbloed MRM, Kamel SM, Koopman CD, Lachaud Q, Mannhardt I, Mol MPH, Mosqueira D, Orlova VV, Passier R, Ribeiro MC, Saleem U, Smith GL, Burton FL, Mummery CL. MUSCLEMOTION: A Versatile Open Software Tool to Quantify Cardiomyocyte and Cardiac Muscle Contraction In Vitro and In Vivo. Circ Res. 2018 Feb 2;122(3):e5-e16. doi: 10.1161/CIRCRESAHA.117.312067.

15. van Meer BJ, Sala L, Tertoolen LGJ, Smith GL, Burton FL, Mummery CL. Quantification of Muscle Contraction In Vitro and In Vivo Using MUSCLEMOTION Software: From Stem Cell-Derived Cardiomyocytes to Zebrafish and Human Hearts. Curr Protoc Hum Genet. 2018 Oct;99(1):e67. doi:10.1002/cphg.67.

16. Lee J, Sutani A, Kaneko R, Takeuchi J, Sasano T, Kohda T, Ihara K, Takahashi K, Yamazoe M, Morio T, Furukawa T, Ishino F. In vitro generation of functional murine heart organoids via FGF4 and extracellular matrix. Nat Commun. 2020 Sep 3;11(1):4283. doi: 10.1038/s41467-020-18031-5.

17. Burridge PW, Matsa E, Shukla P, Lin ZC, Churko JM, Ebert AD, Lan F, Diecke S, Huber B, Mordwinkin NM, Plews JR, Abilez OJ, Cui B, Gold JD, Wu JC. Chemically defined generation of human cardiomyocytes. Nat Methods. 2014 Aug;11(8):855-60. doi:10.1038/nmeth.2999. 
18. Tohyama S, Hattori F, Sano M, Hishiki T, Nagahata Y, Matsuura T, Hashimoto H, Suzuki T, Yamashita H, Satoh Y, Egashira T, Seki T, Muraoka N, Yamakawa H, Ohgino Y, Tanaka T, Yoichi M, Yuasa S, Murata M, Suematsu M, Fukuda K. Distinct metabolic flow enables large-scale purification of mouse and human pluripotent stem cell-derived cardiomyocytes. Cell Stem Cell. 2013 Jan 3;12(1):127-37. doi: 10.1016/j.stem.2012.09.013.

19. Tse G, Lee S, Zhou J, Liu T, Wong ICK, Mak C, Mok NS, Jeevaratnam K, Zhang Q, Cheng SH, Wong WT. Territory-Wide Chinese Cohort of Long QT Syndrome: Random Survival Forest and Cox Analyses. Front Cardiovasc Med. 2021 Feb;5:8:608592. doi:10.3389/fcvm.2021.608592.

20. Bhat HF, Adams ME, Khanday FA. Syntrophin proteins as Santa Claus: role(s) in cell signal transduction. Cell Mol Life Sci. 2013 Jul;70(14):2533-54. doi:10.1007/s00018-012-1233-9.

21. Bhat SS, Ali R, Khanday FA. Syntrophins entangled in cytoskeletal meshwork: Helping to hold it all together. Cell Prolif. 2019 Mar;52(2):e12562. doi:10.1111/cpr.12562.

22. Yang KC, Kyle JW, Makielski JC, Dudley SC Jr. Mechanisms of sudden cardiac death: oxidants and metabolism. Circ Res. 2015 Jun 5;116(12):1937-55. doi: 10.1161/CIRCRESAHA.116.304691.

23. Braunwald $E$. The war against heart failure: the Lancet lecture. Lancet. 2015 Feb 28;385(9970):81224. doi: 10.1016/S0140-6736(14)61889-4.

24. Landstrom AP, Dobrev D, Wehrens XHT. Calcium Signaling and Cardiac Arrhythmias. Circ Res. 2017 Jun 9;120(12):1969-1993. doi: 10.1161/CIRCRESAHA.117.310083.

25. Berra-Romani R, Guerra G, Moccia F. Editorial: Advances and Current Challenges in Calcium Signaling Within the Cardiovascular System. Front Physiol. 2021 Jul 23;12:696315. doi: 10.3389/fphys.2021.696315.

26. Hong T, Shaw RM. Cardiac T-Tubule, Microanatomy. and Function. Physiol Rev. 2017 Jan;97(1):22752. doi:10.1152/physrev.00037.2015.

27. Poulet C, Sanchez-Alonso J, Swiatlowska P, Mouy F, Lucarelli C, Alvarez-Laviada A, Gross P, Terracciano C, Houser S, Gorelik J. Junctophilin-2 tethers T-tubules and recruits functional L-type calcium channels to lipid rafts in adult cardiomyocytes. Cardiovasc Res. 2021 Jan 1;117(1):149-161. doi: $10.1093 / c v r / c v a a 033$.

28. Wang S, Zhou Y, Luo Y, Kan R, Chen J, Xuan H, Wang C, Chen J, Xu T, Li D. SERCA2a ameliorates cardiomyocyte T-tubule remodeling via the calpain/JPH2 pathway to improve cardiac function in myocardial ischemia/reperfusion mice. Sci Rep. 2021 Jan 21;11(1):2037. doi: 10.1038/s41598-02181570-4.

29. Gross P, Johnson J, Romero CM, Eaton DM, Poulet C, Sanchez-Alonso J, Lucarelli C, Ross J, Gibb AA, Garbincius JF, Lambert J, Varol E, Yang Y, Wallner M, Feldsott EA, Kubo H, Berretta RM, Yu D, Rizzo V, Elrod J, Sabri A, Gorelik J, Chen X, Houser SR. Interaction of the Joining Region in Junctophilin-2 With the L-Type $\mathrm{Ca}^{2+}$ Channel Is Pivotal for Cardiac Dyad Assembly and Intracellular $\mathrm{Ca}^{2+}$ Dynamics. Circ Res. 2021 Jan 8;128(1):92-114. doi: 10.1161/CIRCRESAHA.119.315715.

30. Yang Z, Su W, Zhang Y, Zhou L, Xia ZY, Lei S. Selective inhibition of PKC 32 improves Caveolin3 /eNOS signaling and attenuates lipopolysaccharide-induced injury by inhibiting autophagy in $\mathrm{H} 9 \mathrm{C} 2$ 
cardiomyocytes. J Mol Histol. 2021 Aug;52(4):705-715. doi: 10.1007/s10735-021-09990-0.

31. Yang L, Li RC, Xiang B, Li YC, Wang LP, Guo YB, Liang JH, Wang XT, Hou T, Xing X, Zhou ZQ, Ye H, Feng RQ, Lakatta EG, Chai Z, Wang SQ. Transcriptional regulation of intermolecular $\mathrm{Ca}^{2+}$ signaling in hibernating ground squirrel cardiomyocytes: The myocardin-junctophilin axis. Proc Natl Acad Sci U S A. 2021 Apr 6;118(14):e2025333118. doi: 10.1073/pnas.2025333118.

32. Pradhan BS, Prószyński TJ. A Role for Caveolin-3 in the Pathogenesis of Muscular Dystrophies. Int J Mol Sci. 2020 Nov 19;21(22):8736. doi: 10.3390/ijms21228736.

33. Hong TT, Smyth JW, Gao D, Chu KY, Vogan JM, Fong TS, Jensen BC, Colecraft HM, Shaw RM. BIN1 localizes the L-type calcium channel to cardiac T-tubules. PLoS Biol. 2010 Feb 16;8(2):e1000312. doi: 10.1371 /journal.pbio.1000312.

34. Xiao S, Shaw RM. Cardiomyocyte protein trafficking: Relevance to heart disease and opportunities for therapeutic intervention. Trends Cardiovasc Med. 2015 Jul;25(5):379-89. doi:10.1016/j.tcm.2014.12.012.

35. Li J, Richmond B, Hong T. Cardiac T-Tubule cBIN1-Microdomain, a Diagnostic Marker and Therapeutic Target of Heart Failure. Int J Mol Sci. 2021 Feb 25;22(5):2299. doi: 10.3390/ijms22052299.

36. Guo J, Tian Q, Barth M, Xian W, Ruppenthal S, Schaefers HJ, Chen Z, Moretti A, Laugwitz KL, Lipp P. Human BIN1 isoforms grow, maintain and regenerate excitation-contraction couplons in adult rat and human stem cell-derived cardiomyocytes. Cardiovasc Res. 2021 Jun 21:cvab195. doi: 10.1093/cvr/cvab195.

37. Bodi I, Mikala G, Koch SE, Akhter SA, Schwartz A. The L-type calcium channel in the heart: the beat goes on. J Clin Invest. 2005 Dec;115(12):3306-17. doi:10.1172/JCl27167.

38. Novak A, Lorber A, Itskovitz-Eldor J, Binah O. Modeling Catecholaminergic Polymorphic Ventricular Tachycardia using Induced Pluripotent Stem Cell-derived Cardiomyocytes. Rambam Maimonides Med J. 2012 Jul 31;3(3):e0015. doi: 10.5041/RMMJ.10086.

39. Davis FJ, Gupta M, Camoretti-Mercado B, Schwartz RJ, Gupta MP. Calcium/calmodulin-dependent protein kinase activates serum response factor transcription activity by its dissociation from histone deacetylase, HDAC4. Implications in cardiac muscle gene regulation during hypertrophy. J Biol Chem. 2003 May 30;278(22):20047-58. doi: 10.1074/jbc.M209998200.

40. Zhang W, Qi F, Chen DQ, Xiao WY, Wang J, Zhu WZ. $\mathrm{Ca}^{2+} /$ calmodulin-dependent protein kinase Ildelta orchestrates G-protein-coupled receptor and electric field stimulation-induced cardiomyocyte hypertrophy. Clin Exp Pharmacol Physiol. 2010 Aug;37(8):795-802. doi:10.1111/j.14401681.2010.05382.x.

41. Willis BC, Pandit SV, Ponce-Balbuena D, Zarzoso M, Guerrero-Serna G, Limbu B, Deo M, Camors E, Ramirez RJ, Mironov S, Herron TJ, Valdivia HH, Jalife J. Constitutive Intracellular Na+ Excess in Purkinje Cells Promotes Arrhythmogenesis at Lower Levels of Stress Than Ventricular Myocytes From Mice With Catecholaminergic Polymorphic Ventricular Tachycardia. Circulation. 2016 Jun 14;133(24):2348-59. doi: 10.1161/CIRCULATIONAHA.116.021936. 
42. Liu Z, Liu X, Yu H, Pei J, Zhang Y, Gong J, Pu J. Common Variants in TRDN and CALM1 Are Associated with Risk of Sudden Cardiac Death in Chronic Heart Failure Patients in Chinese Han Population. PLoS One. 2015 Jul 21;10(7):e0132459. doi: 10.1371/journal.pone.0132459.

43. Chen H, Valle G, Furlan S, Nani A, Gyorke S, Fill M, Volpe P. Mechanism of calsequestrin regulation of single cardiac ryanodine receptor in normal and pathological conditions. J Gen Physiol. 2013 Aug;142(2):127-36. doi:10.1085/jgp.201311022.

44. Yano K, Zarain-Herzberg A. Sarcoplasmic reticulum calsequestrins: structural and functional properties. Mol Cell Biochem. 1994 Jun 15;135(1):61-70. doi: 10.1007/BF00925961.

45. Wleklinski MJ, Kannankeril PJ, Knollmann BC. Molecular and tissue mechanisms of catecholaminergic polymorphic ventricular tachycardia. J Physiol. 2020 Jul;598(14):2817-34. doi:10.1113/JP276757.

46. Bers DM. Cardiac excitation-contraction coupling. Nature. 2002 Jan 10;415(6868):198-205. doi: 10.1038/415198a.

47. Gray B, Bagnall RD, Lam L, Ingles J, Turner C, Haan E, Davis A, Yang PC, Clancy CE, Sy RW, Semsarian C. A novel heterozygous mutation in cardiac calsequestrin causes autosomal dominant catecholaminergic polymorphic ventricular tachycardia. Heart Rhythm. 2016 Aug;13(8):1652-60. doi:10.1016/j.hrthm.2016.05.004.

48. Josephs K, Patel K, Janson CM, Montagna C, McDonald TV. Compound heterozygous CASQ2 mutations and long-term course of catecholaminergic polymorphic ventricular tachycardia. Mol Genet Genomic Med. 2017 Nov;5(6):788-94. doi:10.1002/mgg3.323.

49. Hoedemaekers YM, Caliskan K, Michels M, Frohn-Mulder I, van der Smagt JJ, Phefferkorn JE, Wessels MW, ten Cate FJ, Sijbrands EJ, Dooijes D, Majoor-Krakauer DF. The importance of genetic counseling, DNA diagnostics, and cardiologic family screening in left ventricular noncompaction cardiomyopathy. Circ Cardiovasc Genet. 2010 Jun;3(3):232-9. doi:10.1161/CIRCGENETICS.109.903898.

50. Kawamura M, Ohno S, Naiki N, Nagaoka I, Dochi K, Wang Q, Hasegawa K, Kimura H, Miyamoto A, Mizusawa Y, Itoh H, Makiyama T, Sumitomo N, Ushinohama H, Oyama K, Murakoshi N, Aonuma K, Horigome H, Honda T, Yoshinaga M, Ito M, Horie M. Genetic Background of Catecholaminergic Polymorphic Ventricular Tachycardia in Japan. Circ J. 2020;84(11):2124-6. doi:10.1253/circj.CJ-660186.

51. Chopra N, Kannankeril PJ, Yang T, Hlaing T, Holinstat I, Ettensohn K, Pfeifer K, Akin B, Jones LR, Franzini-Armstrong C, Knollmann BC. Modest reductions of cardiac calsequestrin increase sarcoplasmic reticulum Ca2+ leak independent of luminal Ca2+ and trigger ventricular arrhythmias in mice. Circ Res. 2007 Sep 14;101(6):617-26. doi: 10.1161/CIRCRESAHA.107.157552.

52. Kalyanasundaram A, Lacombe VA, Belevych AE, Brunello L, Carnes CA, Janssen PM, Knollmann BC, Periasamy M, Gyørke S. Up-regulation of sarcoplasmic reticulum $\mathrm{Ca}(2+)$ uptake leads to cardiac hypertrophy, contractile dysfunction and early mortality in mice deficient in CASQ2. Cardiovasc Res. 2013 May 1;98(2):297-306. doi: 10.1093/cvr/cvs334. 
53. Sherrid MV. Drug Therapy for Hypertrophic Cardiomypathy: Physiology and Practice. Curr Cardiol Rev. 2016;12(1):52-65. doi:10.2174/1573403x1201160126125403.

54. Bairey Merz CN, Pepine CJ, Walsh MN, Fleg JL. Ischemia and No Obstructive Coronary Artery Disease (INOCA): Developing Evidence-Based Therapies and Research Agenda for the Next Decade. Circulation. 2017 Mar 14;135(11):1075-1092. doi: 10.1161/CIRCULATIONAHA.116.024534.

55. Polsinelli VB, Sinha A, Shah SJ. Visceral Congestion in Heart Failure: Right Ventricular Dysfunction, Splanchnic Hemodynamics, and the Intestinal Microenvironment. Curr Heart Fail Rep. 2017 Dec;14(6):519-28. doi:10.1007/s11897-017-0370-8.

56. Jaswal JS, Keung W, Wang W, Ussher JR, Lopaschuk GD. Targeting fatty acid and carbohydrate oxidation--a novel therapeutic intervention in the ischemic and failing heart. Biochim Biophys Acta. 2011 Jul;1813(7):1333-50. doi:10.1016/j.bbamcr.2011.01.015.

57. Sossalla S, Wagner S, Rasenack EC, Ruff H, Weber SL, Schöndube FA, Tirilomis T, Tenderich G, Hasenfuss G, Belardinelli L, Maier LS. Ranolazine improves diastolic dysfunction in isolated myocardium from failing human hearts-role of late sodium current and intracellular ion accumulation. J Mol Cell Cardiol. 2008 Jul;45(1):32-43. doi: 10.1016/j.yjmcc.2008.03.006.

58. Toischer K, Hartmann N, Wagner S, Fischer TH, Herting J, Danner BC, Sag CM, Hund TJ, Mohler PJ, Belardinelli L, Hasenfuss G, Maier LS, Sossalla S. Role of late sodium current as a potential arrhythmogenic mechanism in the progression of pressure-induced heart disease. J Mol Cell Cardiol. 2013 Aug;61:111-22. doi:10.1016/j.yjmcc.2013.03.021.

59. Mosqueira D, Mannhardt I, Bhagwan JR, Lis-Slimak K, Katili P, Scott E, Hassan M, Prondzynski M, Harmer SC, Tinker A, Smith JGW, Carrier L, Williams PM, Gaffney D, Eschenhagen T, Hansen A, Denning C. CRISPR/Cas9 editing in human pluripotent stem cell-cardiomyocytes highlights arrhythmias, hypocontractility, and energy depletion as potential therapeutic targets for hypertrophic cardiomyopathy. Eur Heart J. 2018 Nov 14;39(43):3879-3892. doi: 10.1093/eurheartj/ehy249.

60. Coppini R, Ferrantini C, Yao L, Fan P, Del Lungo M, Stillitano F, Sartiani L, Tosi B, Suffredini S, Tesi C, Yacoub M, Olivotto I, Belardinelli L, Poggesi C, Cerbai E, Mugelli A. Late sodium current inhibition reverses electromechanical dysfunction in human hypertrophic cardiomyopathy. Circulation. 2013 Feb 5;127(5):575-84. doi: 10.1161/CIRCULATIONAHA.112.134932.

\section{Figures}

\section{Figure 1}

Establishment of homozygous SNTA1-deficient hESCs

A. Schematic of the sgRNA designed to the $\mathrm{PH} 1$ region in SNTA1 demonstrates that one adenine nucleotide is inserted into SNTA1 before the PAM sequence. 
B. The DNA molecules of $\mathrm{H} 9$ and H9SNTA1KO cells were detected by DNA sequencing. The DNA was extracted and amplified by PCR. The result showed one adenine nucleotide was inserted into the SNTA1 before the PAM sequence in H9SNTA1KO genome.

C. Utilizing tools from Synthego website to assess the gene editing ratio. The upper graph offered the abstract of indel ratio with the results showing a knockout-score of $99 \%$. The relative contribution of sequence is a nucleotide was inserted before the PAM sequence. The graph below offered the analysis of sequence using the Synthego tools. The red dotted line is under the PAM sequence in control sample diagram. The vertical dotted line demonstrated one adenine inserted in the edited sample diagram. The homozygous SNTA1 knockout hESCs were established.

D. Immunofluorescence staining for pluripotency was performed. Both SSEA4 and NANOG were positive in H9SNTA1KO. SNTA1-knockout did not influence the pluripotency of hESCs.

\section{Figure 2}

H9SNTA1KO differentiated into cardiomyocytes

A. Schematic of hESCs induction into cardiomyocytes using small molecule inhibitors.

B. Image B1 to B3 were hESCs induction into cardiomyocytes using working solutions. Scale bar: $100 \mu \mathrm{m}$. Image B4 showed the mass of beating cardiomyocytes on the $10^{\text {th }}$ day of differentiation. Scale bar: 100 $\mu \mathrm{m}$.

C. The image of $\mathrm{KO}$ exhibited purified by metabolic selection using a glucose-free medium supplemented with lactate. Scale bar: $50 \mu \mathrm{m}$.

D. Immunostaining of TNNT2 (green) and a-actinin (red) in KO. Scale bar: $7.5 \mu \mathrm{m}$.

E. The left graph: Flow cytometry was used to detect a specific cardiac marker, TNNT2. The result demonstrated that the differentiation rate of H9SNTA1KO was similar to the WT without purification. The right graph: Quantification of TNNT2 based on flow cytometry $(n=3)$. ns; not significant, unpaired twosided Student's t test.

F. The left graph: Flow cytometry was used to detect a specific ventricular muscle marker, MYL2. The results demonstrated that the yield of WT and KO was similar purified using metabolic selection. The right graph: Quantification of MYL2 of the flow cytometry $(n=3)$. ns; not significant, unpaired two-sided Student's t test.

Figure 3

Page 19/22 
Structural components increased in KO

A. The left panel: The RNA molecules of WT and KO were extracted and detected using RNA-seq. The results of KEGG pathway enrichment analysis of these genes showed highly enriched in hypertrophic cardiomyopathy on the $30^{\text {th }}$ day of cardiac differentiation.

The right panel: Heatmap showed the discrepancies of genes involved in structural components in WT and $\mathrm{KO}$ as detected by qRT-PCR on the $45^{\text {th }}$ day of cardiac differentiation.

B. Immunostaining of MYL2 (green) in WT and KO and semi-quantification analysis measured on the $45^{\text {th }}$ day of cardiac differentiation. Scale bar: $10 \mu \mathrm{m} . \mathrm{n}=3$ independent experiments. ${ }^{\star} P<0.05$.

C. Immunostaining of TNNT2 (green) in WT and KO and semi-quantification analysis measured on the $45^{\text {th }}$ day of cardiac differentiation. Scale bar: $5 \mu \mathrm{m} . \mathrm{n}=3$ independent experiments. ${ }^{\star \star} P<0.01$.

D. Immunostaining of a-actinin (red) in WT and KO and semi-quantification analysis measured on the $45^{\text {th }}$ day of cardiac differentiation. Scale bar: $5 \mu \mathrm{m} . \mathrm{n}=3$ independent experiments. ${ }^{*} P<0.05$.

\section{Figure 4}

The KO had normal diameter and shorter FPD

A. to $C$. The diameter size of WT and KO were evaluated using flow cytometry on the $15^{\text {th }}, 35^{\text {th }}$, and $45^{\text {th }}$ day of cardiac differentiation.

D. Schematic of the process of microelectrode array (MEA) test.

E. MEA showed the discrepancy of field potential duration (FPD) between WT and KO. $n=9$ independent experiments, ${ }^{\star \star \star \star} P<0.0001$; unpaired two-sided Student's $t$ test.

\section{Figure 5}

The KO exhibited calcium transient abnormality

A. The left panel: The representative line-scan image of WT and KO stained with Fluo-4.

The right panel: Calcium transient profile derived from the left panel.

B. Space-averaged calcium transients showed parameters measured for analysis of calcium handling. 
C. to G. Quantification of peak, time to peak, decay time, contraction duration, and beating rate in WT and

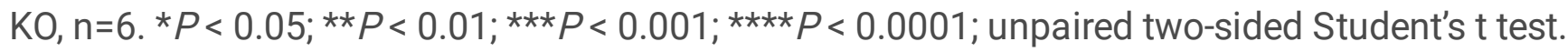

\section{Figure 6}

Abnormal caffeine-evoked $\mathrm{Ca}^{2+}$ release in $\mathrm{KO}$

A. The left panel: The representative line-scan image of caffeine-evoked $\mathrm{Ca}^{2+}$ release in WT and $\mathrm{KO}$ stained with Fluo-4.

The right panel: $\mathrm{Ca}^{2+}$ transients profile induced with $10 \mathrm{mM}$ caffeine in $\mathrm{Ca}^{2+}$-free conditions derived from the left panel.

B. to D. Quantification of peak, time to peak, and decay time in caffeine-evoked $\mathrm{Ca}^{2+}$ transients WT and

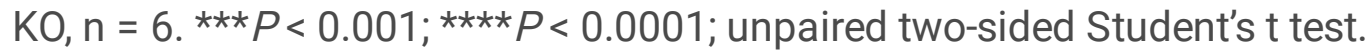

E. Schematic of the process of the contractility assess.

F. Contractility graph of WT and KO.

G. Quantification of contractility in WT and KO based on panel F. $\mathrm{n}=3$ independent experiments. ${ }^{\star \star} P<$ 0.01; unpaired two-sided Student's $t$ test.

\section{Figure 7}

Impairment of calcium handling in KO

A. Heatmap showed the discrepancies about the expression of genes involved in calcium handling in WT and $\mathrm{KO}$.

B.to D. Immunoblot analysis of SERCA2a, BIN1, and CASQ2 in WT and KO.

\section{Figure 8}

Early application of ranolazine improving the calcium handling of $\mathrm{KO}$

A. The left panel: The representative line-scan image of the $K O$ and $K O+R(K O$ treated with $10 \mu \mathrm{M}$ ranolazine) stained with Fluo-4 on the $20^{\text {th }}$ day of cardiac differentiation. 
The right panel: Calcium transient profile derived from the left panel.

B. to $\mathrm{E}$. Quantification of time to peak, peak, contraction duration, and beating rate in $\mathrm{KO}$ and $\mathrm{KO}+\mathrm{R}$ ( $\mathrm{KO}$ treated with $10 \mu \mathrm{M}$ ranolazine), $\mathrm{n}=6 .{ }^{\star \star} P<0.01$; ${ }^{\star \star \star \star} P<0.0001$; unpaired two-sided Student's $\mathrm{t}$ test.

F. Contractility graph of $\mathrm{KO}$ and $\mathrm{KO}+\mathrm{R}$.

G. Quantification of the panel F. $\mathrm{n}=3$ independent experiments. ${ }^{*} P<0.05$. unpaired two-sided Student's $\mathrm{t}$ test.

\section{Figure 9}

Schematic illustrating the disease model of KO

Abnormal calcium handling in $\mathrm{KO}$.

\section{Supplementary Files}

This is a list of supplementary files associated with this preprint. Click to download.

- renamed0ddb5.docx 